\title{
Profiling tear proteomes of patients with unilateral relapsed Behcet's disease-associated uveitis using data-independent acquisition proteomics
}

\author{
Anyi Liang ${ }^{\text {Equal first author, } 1}$, Weiwei Qin ${ }^{\text {Equal first author, } 2,3}$, Meifen Zhang ${ }^{1}$, Fei Gao ${ }^{1}$, Chan Zhao ${ }^{\text {Corresp., } 1}$, Youhe Gao ${ }^{\text {Corresp. } 2}$ \\ 1 Department of Ophthalmology, Peking Union Medical College Hospital, Chinese Academy of Medical Sciences, Beijing, China \\ 2 Department of Biochemistry and Molecular Biology, Gene Engineering Drug and Biotechnology Beijing Key Laboratory, Beijing Normal University, Beijing, \\ China \\ 3 Department of Anesthesiology, Qingdao Municipal Hospital, Qingdao, China \\ Corresponding Authors: Chan Zhao, Youhe Gao \\ Email address: zhaochan@pumch.cn, gaoyouhe@bnu.edu.cn
}

Purpose: To explore whether unilateral relapse of Bechet's disease-associated uveitis (BDU) causes differences in the tear proteome between the diseased and the contralateral quiescent eye and potential tear biomarkers for uveitis recurrence and disease monitoring. Method: To minimize interindividual variations, bilateral tear samples were collected from the same patient $(n=15)$ with unilateral relapse of BDU. A data-independent acquisition (DIA) strategy was used to identify proteins that differed between active and quiescent eyes. Results: A total of 1,797 confident proteins were identified in the tear samples, of which 381 (21.2\%) were also highly expressed in various tissues and organs. Fifty-one (2.8\%) proteins differed in terms of expression between tears in active and quiescent eyes, $9(17.6 \%)$ of which were functionally related to immunity or inflammation. Alpha-1-acid glycoprotein 1 (fold change=3.2, $p=0.007$ ) was increased and Annexin A1 (fold change $=-1.7, p<0.001$ ) was decreased in the tears of the active BDU eye compared to the contralateral quiescent eye.Conclusions: A substantial amount of confident proteins were detected in the tears of BDU patients, including proteins that were deferentially expressed in the uveitis-relapsed eyes and the contralateral quiescent eyes. Some of these identified tear proteins play important roles in immune and inflammatory processes. Tear proteome might be a good source of biomarkers for uveitis. 


\section{Profiling Tear Proteomes of Patients with Unilateral 2 Relapsed Behcet's disease-associated Uveitis Using Data- 3 independent Acquisition Proteomics}

4 Anyi Liang ${ }^{1, \#}$, Weiwei Qin ${ }^{2,3, \# \text {, Meifen Zhang }}{ }^{1}$, Fei Gao ${ }^{1}$, Chan Zhao ${ }^{1, *}$, Youhe Gao ${ }^{2, *}$

$5{ }^{1}$ Department of Ophthalmology, Peking Union Medical College Hospital, Chinese Academy of

6 Medical Sciences, Beijing 100005, China.

$7 \quad 2$ Department of Biochemistry and Molecular Biology, Gene Engineering Drug and

8 Biotechnology Beijing Key Laboratory, Beijing Normal University, Beijing 100875 China

$9 \quad{ }^{3}$ Department of Anesthesiology, Qingdao Municipal Hospital, Qingdao, China.

10 \# Authors contributed equally

11 *Author for correspondence.

Corresponding author 1 :

No.1 Shuaifuyuan Wangfujing Dongcheng District, Beijing, 100730, China

Email address: zhaochan@pumch.cn

Corresponding author 2:

Youhe $\mathrm{Gao}^{2}$

Email address: gaoyouhe@bnu.edu.cn 


\section{Abstract}

26 Purpose: To explore whether unilateral relapse of Bechet's disease-associated uveitis (BDU) causes differences in the tear proteome between the diseased and the contralateral quiescent eye and potential tear biomarkers for uveitis recurrence and disease monitoring.

29

Method: To minimize interindividual variations, bilateral tear samples were collected from the same patient $(n=15)$ with unilateral relapse of BDU. A data-independent acquisition (DIA) strategy was used to identify proteins that differed between active and quiescent eyes.

Results: A total of 1,797 confident proteins were identified in the tear samples, of which 381 $(21.2 \%)$ were also highly expressed in various tissues and organs. Fifty-one $(2.8 \%)$ proteins differed in terms of expression between tears in active and quiescent eyes, 9 (17.6\%) of which were functionally related to immunity or inflammation. Alpha-1-acid glycoprotein 1 (fold change $=3.2, \mathrm{p}=0.007$ ) was increased and Annexin A1 (fold change $=-1.7, \mathrm{p}<0.001$ ) was decreased in the tears of the active BDU eye compared to the contralateral quiescent eye.

Conclusions: A substantial amount of confident proteins were detected in the tears of BDU patients, including proteins that were deferentially expressed in the uveitis-relapsed eyes and the contralateral quiescent eyes. Some of these identified tear proteins play important roles in immune and inflammatory processes. Tear proteome might be a good source of biomarkers for uveitis.

Keywords: Behcet's disease, biomarkers, intraocular inflammation, tear proteomics, uveitis 


\section{Introduction}

Tear fluid is a complex mixture of water, electrolytes, metabolites, lipids and proteins (mucins, enzymes, glycoproteins, immunoglobulins and others) secreted mainly by the main and accessory lacrimal glands ${ }^{1,2}$. Tears can be easily and noninvasively accessed ${ }^{3}$ and are considered a useful source for biomarker research of ocular and systemic diseases. According to a recent review, hundreds of potential specific molecular biomarkers in tears were found to be associated with ocular diseases, including dry eye disease, keratoconus and thyroid associated orbitopathy. Other reports showed that tears can reflect the state of breast cancer, prostate cancer and multiple sclerosis $^{2,4,5}$. Moreover, tears may reflect central metabolism in some neurological disorders ${ }^{6-9}$.

Behcet's disease (BD) is a chronic, relapsing systemic inflammatory disease characterized by recurrent oral aphthae, ocular involvement and a variety of other clinical features. Bechet's disease-associated uveitis (BDU) involves about half of the BD patients and is the most common cause of morbidity ${ }^{10,11}$. Recurrent attacks of BDU are common and accumulating damages to the fundus structures frequently leads to severe vision loss or even blindness. Unfortunately, recurrence of BDU is usually abrupt and rarely heralded by prodromes or warning signs, and signs of active disease are often obscured by complications involving the refractive media such as cataract, pupillary block, etc. Therefore, there is an urgent need to discover biomarkers from easily accessible biofluids such as tears, to monitor disease activity and to predict recurrence in BDU patients.

Interestingly, despite being a symmetrical organ, the eyes of BDU patients with unilateral uveitis relapse show dramatically distinct clinical manifestations. While the quiescent eye appears completely normal, the contralateral active eye might have severe visual acuity impairment possibly caused by anterior uveitis, vitritis, retinal vasculitis, retinitis, cystoid macular edema or papillitis ${ }^{12}$. It is possible that, considering the anatomical proximity, intraocular inflammation may cause substantial changes in the tear proteome through local mechanisms, making tears a potential resource of biomarkers for the actively inflammatory eye. To address this hypothesis and to minimize interindividual variations, tear samples were simultaneously collected from both eyes of BDU patients with unilateral uveitis relapse. Samples were preserved by our previously developed "dry" method ${ }^{13}$ and further analyzed by a dataindependent acquisition (DIA) strategy. We aimed to explore whether unilateral relapse of BDU causes differences in the tear proteome between the diseased and the contralateral quiescent eye and to identify potential tear biomarkers for uveitis recurrence and disease monitoring.

\section{Materials \& Methods}


A summary of the overall experimental approach is presented in Fig.1.

\section{Patients}

BDU patients presenting to our center with acute unilateral relapse of panuveitis between January 2018 and June 2018 were included. The consent procedure and the study protocol were approved by the Institutional Review Board of the Institute of Basic Medical Sciences, Chinese Academy of Medical Sciences. (Project No. 007-2014). Written informed consent was obtained from each subject prior to the study.

Patients were diagnosed with BD according to the International Study Group (ISG) or International Criteria for Behcet's Disease (ICBD) ${ }^{14}$. Acute relapse of panuveitis was defined as a decrease in visual acuity and the presence of a combination of anterior uveitis (more than $0.5+$ cells in the anterior chamber), vitritis (more than 1+ cells) and inflammation of the posterior segment with the presence of at least one of the following: retinal vasculitis, retinitis, cystoid macular edema and papillitis ${ }^{12}$. The contralateral eye was reported to be quiescent for at least two months. Patients with any of the following conditions were excluded: 1) other ocular or orbital diseases, including allergic conjunctivitis, infectious conjunctivitis, keratitis of infectious or noninfectious cause, thyroid associated orbitopathy, primary open angle glaucoma and primary angle closure glaucoma; 2) previous ocular trauma or surgeries; 3 ) other forms of uveitis; 4) other systemic diseases, such as diabetes, hypertension, cardiovascular diseases, neurological disorders and irrelevant rheumatic diseases; and 5) a Schirmer test of less than $10 \mathrm{~mm}$ in either eye.

\section{Tear sample collection and preservation}

Tear samples were collected and preserved using a novel Schirmer strip-based dry method ${ }^{13}$. In detail, Schirmer strips (Tianjin Jingming New Technological Devepoment Co.,Ltd) were placed at the lateral $1 / 3$ of the lower conjunctival sacs of both eyes for $5 \mathrm{~min}$, and strips with tears exceeding $10 \mathrm{~mm}$ were collected. The Schirmer strips were dried immediately with a hair dryer $\left(70^{\circ} \mathrm{C}\right.$ for $\left.1 \mathrm{~min}\right)$ and were stored in properly labeled vacuum-sealed bags at room temperature and transferred to $-80^{\circ} \mathrm{C}$ freezer within 2 weeks.

\section{Sample preparation for mass spectrometry}

Tear protein extraction: The strip was cut into small pieces and transferred to a $0.6 \mathrm{~mL}$ tube. Next, $200 \mu \mathrm{L}$ elution buffer $\left(100 \mathrm{mM} \mathrm{NH} \mathrm{H}_{3} \mathrm{HCO}_{3}, 50 \mathrm{mM} \mathrm{NaCl}\right)$ was added and gently shaken for $2 \mathrm{~h}$ at room temperature. The tube was punctured at the bottom with a cannula, placed in a larger tube $(1.5 \mathrm{~mL})$ and centrifuged at $12,000 \mathrm{~g}$ for $5 \mathrm{~min}^{15}$. The filtrate in the outer tube was collected and quantified by the Bradford method.

Tryptic digestion: The proteins were digested with trypsin (Promega, USA) using filteraided sample preparation methods ${ }^{16}$. Briefly, $200 \mu \mathrm{g}$ of the protein sample was loaded on the 10- 
$\mathrm{kD}$ filter unit (Pall, USA). For digestion, the protein solution was reduced with $4.5 \mathrm{mM}$ DTT for $1 \mathrm{~h}$ at $37^{\circ} \mathrm{C}$ and then alkylated with $10 \mathrm{mM}$ of indoleacetic acid for $30 \mathrm{~min}$ at room temperature in the dark. Finally, the proteins were digested with $3 \mu \mathrm{g}$ of trypsin for $14 \mathrm{~h}$ at $37^{\circ} \mathrm{C}$. The peptides were digested using Oasis HLB cartridges (Waters, USA). The resulting peptides were dried and desalted in a SpeedVac (Thermo Fisher Scientific, Waltham, MA). The dried peptide samples were resuspended in $0.1 \%$ formic acid and were quantified using a PierceTM BCA protein assay kit. Two micrograms of each sample were loaded for LC-MS/MS analysis using a data-independent acquisition (DIA) method.

High-pH fractionation: Equal volumes of tryptic digested peptides of each sample were pooled to generate the pooled sample (100 $\mu \mathrm{g}$ of peptides) for the development of a spectral library and for DIA analysis optimization and quality control. Sixty micrograms of pooled peptides was then fractionated using a high-pH spin column (Thermo Pierce, USA). After equilibration of the column, the dried sample was resuspended in $100 \%$ Buffer A (acetonitrile: $\mathrm{H}_{2} \mathrm{O}, 90: 10$ with $0.1 \%$ formic acid and $10 \mathrm{mM}$ of ammonium formate) and loaded onto the column, then eluted with Buffer B at 5, 7.5, 10, 12.5, 15, 17.5, 20, 30, 50 and 70\% of Buffer B $\left(\mathrm{H}_{2} \mathrm{O}\right.$ with $0.1 \%$ formic acid). Fractionated samples were dried completely and resuspended in $20 \mu \mathrm{l}$ of $0.1 \%$ formic acid. Six microliters of each of the fractions was loaded for LC-MS/MS analysis using a data-dependent acquisition (DDA) method.

\section{LC-MS/MS setup for data-dependent and data-independent acquisition}

LC-MS/MS data acquisition was performed on a Fusion Lumos mass spectrometer (Thermo Fisher Scientific) interfaced with an EASY-nLC 1,000 UHPLC system (Thermo Fisher Scientific). For both DDA and DIA analyses, the same LC settings were used for retention time stability. For facilitating retention time alignments among samples, a retention time kit (iRT kit from Biognosys, Switzerland) was spiked at a concentration of 1:20 v/v in all samples ${ }^{17}$. The digested peptides were dissolved in $0.1 \%$ formic acid and loaded on a trap column $(75 \mu \mathrm{m} \times 2$ $\left.\mathrm{cm}, 3 \mu \mathrm{m}, \mathrm{C} 18,100 \mathrm{~A}^{\circ}\right)$. The eluent was transferred to a reversed-phase analytical column (50 $\left.\mu \mathrm{m} \times 500 \mathrm{~mm}, 2 \mu \mathrm{m}, \mathrm{C} 18,100 \mathrm{~A}^{\circ}\right)$. The eluted gradient was $5-30 \%$ buffer $\mathrm{B}(0.1 \%$ formic acid in $99.9 \%$ acetonitrile; flow rate of $0.6 \mu \mathrm{l} / \mathrm{min}$ ) for $60 \mathrm{~min}$.

To acquire a spectral library for use in DIA data extraction, $6 \mu 1$ of each of the fractions was analyzed by data-dependent acquisition. The full scan was done with a $60 \mathrm{~K}$ resolution at $200 \mathrm{~m} / \mathrm{z}$ from 350 to $1,500 \mathrm{~m} / \mathrm{z}$ with an AGC target of $1 \mathrm{e} 6$ and a max injection time of $50 \mathrm{~ms}$. Monoisotopic masses were then selected for further fragmentation for ions with a 2 to 6 positive charge within a dynamic exclusion range of 30 seconds and a minimum intensity threshold of 1e4 ions. Precursor ions were isolated using the quadrupole with an isolation window of $1.6 \mathrm{~m} / \mathrm{z}$. The most intense ions per survey scan (top speed mode) were selected for collision-induced dissociation (CID) fragmentation, and the resulting fragments were analyzed in the Orbitrap with the resolution set to 60,000. The normalized collision energy for higher energy collision dissociation (HCD)-MS2 experiments was set to $32 \%$, the AGC target was set at 5e4 and the 
152

153

154

155

156

157

158

159

160

161

162

163

164

165

166

167

168

169

170

171

172

173

174

175

176

177

178

179

180

181

182

183

184

185

186

187

maximum injection time was set to $30 \mathrm{~ms}$. The DDA cycle was limited to 3 seconds.

For data-independent acquisition, two $\mu \mathrm{g}$ of each sample was analyzed. Survey MS scans were acquired in the Orbitrap using a range of 350-1,550 m/z with a resolution of 120,000 at 200 $\mathrm{m} / \mathrm{z}$. The AGC target was set at $1 \mathrm{e} 6$ with a $50 \mathrm{~ms}$ max injection time. Twenty-four optimal acquisition windows covered a mass range from 350 to $1,500 \mathrm{~m} / \mathrm{z}$ (Supplemental Table S1). The normalized collision energy for HCD-MS2 experiments was set to $32 \%$, the AGC target was set at $2 \mathrm{e} 5$ and the maximum injection time was set to $54 \mathrm{~ms}$. A quality control DIA analysis of the pooled sample was inserted after every ten tear samples were tested.

\section{Data analysis}

The raw MS data files acquired by the DDA mode for library construction were processed using Proteome Discoverer (version 2.1; Thermo Fisher Scientific, USA) with SEQUEST HT against the SwissProt human database (released in July 2016, containing 20,228 sequences) and the Biognosys iRT peptides sequences. SEQUEST HT Search parameters consisted of the parent ion mass tolerance, $10 \mathrm{ppm}$; fragment ion mass tolerance, $0.02 \mathrm{Da}$; fixed modifications, carbamidomethylated cysteine (+58.00 Da); and variable modifications, oxidized methionine $(+15.995 \mathrm{Da})$. Other settings included the default parameters. All identified proteins had a false discovery rate (FDR) of $\leq 1 \%$. To generate the spectral libraries, DDA spectra were analyzed as described above, and a spectral library was generated using the spectral library generation functionality of Spectronaut Pulsar (Biognosys, Switzerland). The library was devised by importing the search results and the raw files using Spectronaut with the default parameters.

The raw DIA files were imported to Spectronaut Pulsar, and the default settings were used for targeted analysis. In brief, a dynamic window for the XIC extraction window and a nonlinear iRT calibration strategy were used. Mass calibration was set to local mass calibration. Interference correction on the MS1 and MS2 levels was enabled, removing fragments/isotopes from quantification based on the presence of interfering signals but keeping at least three for quantification. The FDR was set to $1 \%$ at the peptide precursor level and at $1 \%$ at the protein level. The significance criteria for a $T$-test was a $\mathrm{p}$ value $<0.01$. A minimum of two peptides matched to a protein and a fold change $>1.5$ were used as the criteria for the identification of differentially expressed proteins.

\section{Results}

\section{Clinical characteristics of BDU patients}

To minimize interindividual variations, bilateral tear samples were collected from the same BDU patient $(n=15)$ with unilateral uveitis relapse. The average age of the patients was 28.6 years old, and the female-to-male ratio was 1:4. Seven and eight patients had active inflammation in the left and right eye, respectively. The amounts of each tear samples collected by the Schirmer strips were between 10 and $30 \mathrm{~mm}$ (Table 1). 


\section{Spectral library establishment and DIA method optimization}

The spectral library that was generated, as described in the methods, contained 16,135 peptides corresponding to 2,779 protein groups. Prior to individual sample analysis, the pooled peptide sample was subjected to DIA experiments in order to refine the acquisition windows list and the cycle time (data points per peak). Ultimately, 25 variable windows were used (Supplemental Table S1), resulting in 7-8 data points per peak (cycle time of $1.8 \mathrm{~s}$ ). A quality control DIA analysis of the pooled sample was inserted after every 10 tear samples were tested. The number of proteins with relative abundance CV below 30\% was 1,184 (85\%) (Supplemental Table S2), suggesting a good reproducibility of our study.

\section{Tear proteome analysis}

\subsection{Tear proteome profile identified from BDU patients}

With the advancement of proteomic techniques, two recent studies were able to detect more than 1,500 tear proteins (International Protein Index (IPI) protein human database version 3.76 and UniProtKB human database were used respectively) ${ }^{16}{ }^{18}$. The comparison and number of overlapped proteins among the 3 studies was shown in Fig.2, and it revealed relatively high inter-study variations. In the current study, 1,797 confident proteins were identified in the 30 tear samples from both eyes of the 15 patients (Supplemental Table S3). There were totally 418 $(23.3 \%)$ proteins in our study that were also detected in the other 2 studies, with $298(16.6 \%)$ common proteins in Zhou L's and 159 (8.8\%) in Ass C's.

We also compared the proteins detected in our study with the tissue-enriched proteome ${ }^{19}$. In all, $381(21.2 \%)$ proteins identified in the tear samples were also highly expressed in various tissues and organs. This number is comparable to the value from our previous tear proteome study which identified 365 common proteins (out of $514,71.0 \%$ ) ${ }^{13}$.

\subsection{Tear proteome variation between different disease statuses}

All tear samples were assigned to either the active (A) or the quiescent (Q) group according to the inflammatory status of the eye (Table 1). Differential proteins were screened with the following criteria: fold change $\geq 1.5$ between the two groups and a P-value of paired t-test $<0.05$. The p-values were adjusted by Benjamini \& Hochberg method. A total of $51(2.8 \%)$ differential proteins were identified (details are described in Supplemental Table S4), among which 9 $(17.6 \%)$ of them were reported to be involved in immunity (Table 2).

\section{Discussion}

In this study, protein profiles in the tears of BDU patients were analyzed by data-independent acquisition proteomics and compared between the actively-uveitic and the contralateral quiescent eye. In total 1,797 tear proteins were detected, 51 of which were found to be differentially 
222

223

224

225

226

227

228

229

230

231

232

233

234

235

236

237

238

239

240

241

242

243

244

245

246

247

248

249

250

251

252

253

254

255

256

257

258

259

expressed in the inflammatory and quiet eyes. Some of these differential proteins were reported to be related to immunity or inflammation.

For comparison of tear protein lists from these Zhou L's, Ass C's and our study, we downloaded the raw data (a list of detailed protein profiles with protein name, IPI number or entry number) of the 2 papers and searched them in the corresponding database. Then we transformed all the proteins from the 3 lists to a comparable form, either protein name or entry number, to make the comparison. The inter-study variability of tear protein profiles suggests that different devices, techniques and databases might yield highly different detection results. On the other hand, the vast majorities of the tear proteins are physiologically redundant and may be influenced by a variety of genetic, environmental, local and systemic factors. While the great magnitude of variability poses challenges to tear proteomic studies, the potential of tear proteome to reflect a variety of environmental and physiological conditions also makes it a valuable resource of biomarker. The strength of our current study is that tear samples were simultaneously collected from both eyes of BDU patients with unilateral uveitis relapse, which theoretically can greatly reduce sources of variabilities including environmental, interindividual and time-dependent variations.

Noticeably, more than 300 proteins identified in our tear samples were also highly expressed in various tissues and organs. There are 2 possible sources of these common proteins, either constitutively expressed in eyes and excreted into tears, or come from distant organs or tissues which release these proteins into the blood circulation, and reach in tears. If it is the former, then the changes of these proteins might reflect the status of the eyes, either in physiological or pathological conditions, for example, in uveitis, and can be used to monitor ocular diseases. If the latter is true, then it further validates findings in previous studies that tear might be a good window for monitoring the change of these tissues or organs.

Fifty-one differential proteins were identified between the uveitic and contralateral quiescent eye. Notably, Alpha-1-acid glycoprotein 1 ( $\alpha 1$-AGP), an acute phase protein produced in reaction to systemic inflammation ${ }^{20,21}$, was increased in active BDU. This immunomodulatory protein was found to increase in serum concentration and its glycosylation would experienced qualitative changes in inflammatory status ${ }^{22,23}$. On the other hand, Annexin A1 (ANXA1) ${ }^{24-27}$, a protein found to be anti-inflammatory, was expressed less in the tears of the active uveitic eye.

Interestingly, 2 immunity related proteins detected to change in our study were also reported to decrease or increase in dry eye disease (DE) 28 , namely ANXA1 and Lysozyme (LYZ). Noticeably, while LYZ decreased in both DE and active BDU, ANXA1 changed in the opposite directions in these 2 diseases. Interestingly, although LYZ was formerly known to play a role in driving a pro-inflammatory response, it is also found to have inflammation limiting and immunedampening effects ${ }^{29}$. So it's not surprising that LYZ was reduced in both active BDU and DE. Contradictorily, ANXA1 increased in DE while decreased in active BDU. ANXA1 plays multifunctional roles in innate and adaptive immunity, and is well-known for its inflammation 
260

261

262

263

264

265

266

267

268

269

270

271

272

273

274

275

276

277

278

279

280

281

282

283

284

285

286

287

288

resolution property. The decrease of ANXA1 in tears from BDU possibly implies loss of inflammation homeostasis and an actively-inflammed status in active uveitis ${ }^{30-32}$. But the mechanism of increase of ANXA1 in DE needs further explorations. Therefore tear proteins such as $\alpha 1$-AGP and ANXA1 were promising tear biomarkers for uveitis monitoring.

Candidate proteins validation, either by immunological or MS methods, is an important step in studies of biomarkers discovery. To the best of our knowledge, MS methods, multiple reaction monitoring and parallel reaction monitoring (MRM/PRM)-based approaches, are a favorable alternative to immunoassays for quantitative measurement of proteins. They are not necessarily dependent on the use of antibodies and can therefore be rapidly and cost-efficiently developed in comparison to traditional ELISAs. These advantages led many scientists to propose that affinitybased methods for protein quantification, such as ELISA or Western blot, will be soon replaced by mass spectrometric strategies ${ }^{33-35}$. So it makes more sense to validate these changed proteins in tear samples by MRM/PRM-based proteomics strategy. However, the collection of clinical tear samples was time-consuming, so we are still working on collecting more tear samples from uveitis patients including active BDU patients, quiescent BDU patients, active Vogt-KoyanagiHarada (VKH) patients and quiescent VKH patients and carrying out further validation experiments.

This study was a primary exploratory experiment that was limited by a relatively small sample size. Enlarging the samples and enriching the grouping strategies will yield a more clinically applicable result. As was mentioned above, another limitation of our study was that the candidate biomarkers were not validated with MS based-methods. Further validation studies are needed to ensure the accuracy and reliability of the candidate biomarkers.

\section{Conclusion}

In this study, 1,797 confident proteins were detected in the tears of BDU patients, among which 51 differential proteins were identified between the uveitis-relapsed eyes and the contralateral quiescent eyes. Some of these identified tear proteins play important roles in immune and inflammatory processes, with $\alpha 1$-AGP and ANXA1 being promising tear biomarkers for BDU monitoring. Tear proteomes might be a good source of biomarkers for uveitis. 


\section{Acknowledgements}

290 National Key Research and Development Program of China (2018YFC0910202, 291 2016YFC1306300), Beijing Natural Science Foundation (7172076), Beijing cooperative 292 construction project (110651103), Beijing Normal University (11100704), 2016 PUMCH 293 Science Fund for Junior Faculty (pumch-2016-2.27).

\section{Conflict of interest statement}

295 The authors declare that they have no competing interests.

296

297 Supporting information

298 The raw mass spectrometric files were stored at the public repository iProX (URL: 299 https:/www.iprox.org/page/PSV023.html;?url=1584623845406Ia8E, passwords: L7Rh)

300

301

302

303

304 
306

307

308

309

310

311

312

313

314

315

316

317

318

319

320

321

322

323

324

325

326

327

328

329

330

331

332

333

1. Azkargorta M, Soria J, Acera A, Iloro I, Elortza F. Human tear proteomics and peptidomics in ophthalmology: Toward the translation of proteomic biomarkers into clinical practice. J Proteomics. 150, 359-367 (2017).

2. Pieragostino D, D'Alessandro M, di Ioia M, Di Ilio C, Sacchetta P, Del Boccio P. Unraveling the molecular repertoire of tears as a source of biomarkers: beyond ocular diseases. Proteomics Clin Appl. 9(1-2), 169-86 (2015).

3. Quah JH, Tong L, Barbier S. Patient acceptability of tear collection in the primary healthcare setting. Optom Vis Sci. 91(4), 452-8 (2014).

4. Hagan S, Martin E, Enríquez-de-Salamanca A. Tear fluid biomarkers in ocular and systemic disease: potential use for predictive, preventive and personalised medicine. EPMA J. 7, 15 (2016).

5. von TUHN, Funke S, Grus FH. Tears as a source of biomarkers for ocular and systemic diseases. Exp Eye Res. 117, 126-37 (2013).

6. Coyle PK, Sibony P, Johnson C. Oligoclonal IgG in tears. Neurology. 37(5), 853-6 (1987).

7. Lolli F, Franciotta D. Oligoclonal bands in tears. Mult Scler. 16(6), 760; author reply 761 (2010).

8. Lebrun C, Forzy G, Collongues N, Cohen M, de Seze J, Hautecoeur P. Tear analysis as a tool to detect oligoclonal bands in radiologically isolated syndrome. Rev Neurol (Paris). 171(4), 390-3 (2015).

9. Calais G, Forzy G, Crinquette C, Mackowiak A, de Seze J, Blanc F, Lebrun C, Heinzlef O, Clavelou P, Moreau T, Hennache B, Zephir H, Verier A, Neuville V, Confavreux C, Vermersch $\mathrm{P}$, Hautecoeur P. Tear analysis in clinically isolated syndrome as new multiple sclerosis criterion. Mult Scler. 16(1), 87-92 (2010).

10. Davatchi F, Chams-Davatchi C, Shams H, Shahram F, Nadji A, Akhlaghi M, Faezi T, Ghodsi Z, Sadeghi Abdollahi B, Ashofteh F, Mohtasham N, Kavosi H, Masoumi M. Behcet's disease: epidemiology, clinical manifestations, and diagnosis. Expert Rev Clin Immunol. 13(1): 57-65 (2017). 
334 11. You J, Willcox MD, Madigan MC, Wasinger V, Schiller B, Walsh BJ, Graham PH, 335 Kearsley JH, Li Y. Tear fluid protein biomarkers. Adv Clin Chem. 62, 151-96 (2013).

336 12. Markomichelakis N, Delicha E, Masselos S, Fragiadaki K, Kaklamanis P, Sfikakis PP. A single infliximab infusion vs corticosteroids for acute panuveitis attacks in Behçet's disease: a comparative 4-week study. Rheumatology (Oxford). 50(3), 593-7 (2011).

13. Qin W, Zhao C, Zhang L, Wang T, Gao Y. A Dry Method for Preserving Tear Protein Samples. Biopreserv Biobank. 15(5), 417-421 (2017).

14. Criteria for diagnosis of Behçet's disease. International Study Group for Behçet's Disease. Lancet. 335(8697), 1078-80 (1990).

15. Posa A, Bräuer L, Schicht M, Garreis F, Beileke S, Paulsen F. Schirmer strip vs. capillary tube method: non-invasive methods of obtaining proteins from tear fluid. Ann Anat. 195(2), 137-42 (2013).

16. Aass C, Norheim I, Eriksen EF, Thorsby PM, Pepaj M. Single unit filter-aided method for fast proteomic analysis of tear fluid. Anal Biochem. 480, 1-5 (2015).

17. Escher C, Reiter L, MacLean B, Ossola R, Herzog F, Chilton J, MacCoss MJ, Rinner O. Using iRT, a normalized retention time for more targeted measurement of peptides. Proteomics. 12(8), 1111-21 (2012).

18. Zhou L, Zhao SZ, Koh SK, Chen L, Vaz C, Tanavde V, Li XR, Beuerman RW. In-depth analysis of the human tear proteome. J Proteomics. 75(13), 3877-85 (2012).

19. Uhlén M, Fagerberg L, Hallström BM, Lindskog C, Oksvold P, Mardinoglu A, Sivertsson Å, Kampf C, Sjöstedt E, Asplund A, Olsson I, Edlund K, Lundberg E, Navani S, Szigyarto CA, Odeberg J, Djureinovic D, Takanen JO, Hober S, Alm T, Edqvist PH, Berling H, Tegel H, Mulder J, Rockberg J, Nilsson P, Schwenk JM, Hamsten M, von Feilitzen K, Forsberg M, Persson L, Johansson F, Zwahlen M, von Heijne G, Nielsen J, Pontén F. Proteomics. Tissue-based map of the human proteome. Science. 347(6220), 1260419 (2015).

20. Fitos I, Visy J, Zsila F, Mády G, Simonyi M. Selective binding of imatinib to the genetic variants of human alpha1-acid glycoprotein. Biochim Biophys Acta. 1760(11), 1704-12 (2006).

21. Zsila F, Iwao Y. The drug binding site of human alpha1-acid glycoprotein: insight from 
364

365

366

367

368

369

370

371

372

373

374

375

376

377

378

379

380

381

382

383

384

induced circular dichroism and electronic absorption spectra. Biochim Biophys Acta. 1770(5), 797-809 (2007).

22. Ceciliani F, Lecchi C. The Immune Functions of $\alpha 1$ Acid Glycoprotein. Curr Protein Pept Sci. 20(6), 505-524 (2019).

23. Shiyan SD, Bovin NV. Carbohydrate composition and immunomodulatory activity of different glycoforms of alpha1-acid glycoprotein. Glycoconj J. 14(5), 631-638 (1997).

24. Arcone R, Arpaia G, Ruoppolo M, Malorni A, Pucci P, Marino G, Ialenti A, Di Rosa M, Ciliberto G. Structural characterization of a biologically active human lipocortin 1 expressed in Escherichia coli. Eur J Biochem. 211(1-2), 347-55 (1993).

25. Leoni G, Neumann PA, Kamaly N, Quiros M, Nishio H, Jones HR, Sumagin R, Hilgarth RS, Alam A, Fredman G, Argyris I, Rijcken E, Kusters D, Reutelingsperger C, Perretti M, Parkos CA, Farokhzad OC, Neish AS, Nusrat A. Annexin A1-containing extracellular vesicles and polymeric nanoparticles promote epithelial wound repair. $J$ Clin Invest. 125(3), 1215-27 (2015).

26. Ernst S, Lange C, Wilbers A, Goebeler V, Gerke V, Rescher U. An annexin 1 N-terminal peptide activates leukocytes by triggering different members of the formyl peptide receptor family. J Immunol. 172(12), 7669-76 (2004).

27. D'Acquisto F, Merghani A, Lecona E, Rosignoli G, Raza K, Buckley CD, Flower RJ, Perretti M. Annexin-1 modulates T-cell activation and differentiation. Blood. 109(3), 1095-102 (2007).

28. Ragland SA, Criss AK. From bacterial killing to immune modulation: Recent insights into the functions of lysozyme. PLoS Pathog. 13(9), e1006512 (2017).

29. Willcox M, Argüeso P, Georgiev GA, Holopainen JM, Laurie GW, Millar TJ, Papas EB, Rolland JP, Schmidt TA, Stahl U, Suarez T, Subbaraman LN, Uçakhan OÖ, Jones L. TFOS DEWS II Tear Film Report. Ocul Surf. 15(3): 366-403 (2017).

30. Bruschi M, Petretto A, Vaglio A, Santucci L, Candiano G, Ghiggeri GM. Annexin A1 and Autoimmunity: From Basic Science to Clinical Applications. Int J Mol Sci. 19(5) (2018).

31. Gobbetti T, Cooray SN. Annexin A1 and resolution of inflammation: tissue repairing properties and signalling signature. Biol Chem. 397(10), 981-993 (2016). 
394 32. Sugimoto MA, Vago JP, Teixeira MM, Sousa LP. Annexin A1 and the Resolution of 395 Inflammation: Modulation of Neutrophil Recruitment, Apoptosis, and Clearance. $J$ $396 \quad$ Immunol Res. 2016, 8239258 (2016).

397 33. Aebersold R, Burlingame AL, Bradshaw RA. Western blots versus selected reaction 398 monitoring assays: time to turn the tables. Mol Cell Proteomics. 12(9): 2381-2 (2013).

399

400

401

402

403

404

405

406

407
34. Mermelekas G, Vlahou A, Zoidakis J. SRM/MRM targeted proteomics as a tool for biomarker validation and absolute quantification in human urine. Expert Rev Mol Diagn. 15(11): 1441-54 (2015).

35. Mann M. Can proteomics retire the western blot. J Proteome Res. 7(8): 3065 (2008).

36. Zoubeidi A, Ettinger S, Beraldi E, Hadaschik B, Zardan A, Klomp LW, Nelson CC, Rennie PS, Gleave ME. Clusterin facilitates COMMD1 and I-kappaB degradation to enhance NF-kappaB activity in prostate cancer cells. Mol Cancer Res. 8(1), 119-30 (2010). 


\section{Table $\mathbf{1}$ (on next page)}

Demographic characteristics, inflammatory status and amount of tears collected from each eye of the enrolled BDU patients

A: active (acute relapse of panuveitis); Q: quiescent (stable for at least two months).

There was no statistically significant difference of tear amounts between the active and the quiescent eyes $(-0.27 m m \pm 2.34 m m, p=0.934)$. 
1 Table 1. Demographic characteristics, inflammatory status and amount of tears collected

2 from each eye of the enrolled BDU patients

\begin{tabular}{|c|c|c|c|c|c|c|}
\hline \multirow[t]{2}{*}{ Number } & \multirow[t]{2}{*}{ Sex } & \multirow[t]{2}{*}{ Age } & \multicolumn{2}{|c|}{ Right eye } & \multicolumn{2}{|c|}{ Left eye } \\
\hline & & & Status & $\begin{array}{l}\text { Amount } \\
(\mathrm{mm})\end{array}$ & Status & $\begin{array}{l}\text { Amount } \\
(\mathrm{mm})\end{array}$ \\
\hline 1 & $\mathrm{M}$ & 23 & A & 20 & Q & 30 \\
\hline 2 & M & 30 & Q & 22.5 & A & 30 \\
\hline 3 & $\mathrm{~F}$ & 26 & Q & 15 & A & 30 \\
\hline 4 & M & 28 & Q & 25 & A & 25 \\
\hline 5 & M & 26 & A & 10 & Q & 12.5 \\
\hline 6 & $\mathrm{~F}$ & 32 & A & 12 & Q & 14 \\
\hline 7 & $\mathrm{M}$ & 18 & A & 25 & Q & 25 \\
\hline 8 & $\mathrm{M}$ & 28 & A & 15 & Q & 20 \\
\hline 9 & $\mathrm{~F}$ & 29 & $\mathrm{Q}$ & 30 & A & 25 \\
\hline 10 & $\mathrm{M}$ & 28 & A & 10 & Q & 15 \\
\hline 11 & $\mathrm{M}$ & 22 & $\mathrm{Q}$ & 12 & A & 13 \\
\hline 12 & $\mathrm{M}$ & 34 & Q & 23 & A & 20 \\
\hline 13 & M & 25 & A & 17 & Q & 23 \\
\hline 14 & M & 33 & A & 27 & Q & 20 \\
\hline 15 & M & 48 & $Q$ & 18 & A & 22 \\
\hline
\end{tabular}

3 A: active (acute relapse of panuveitis); Q: quiescent (stable for at least two months).

4 There was no statistically significant difference of tear amounts between the active and the 5 quiescent eyes $(-0.27 \mathrm{~mm} \pm 2.34 \mathrm{~mm}, \mathrm{p}=0.934)$.

6

7 


\section{Table 2 (on next page)}

Details of the 9 differential proteins related to immunity

* a positive fold change indicates increased protein concentration in the relapsed eye compared to the contralateral quiescent eye; a negative fold change indicates decreased protein concentration in the relapsed eye compared to the contralateral quiescent eye 
Table 2. Details of the 9 differential proteins related to immunity

\begin{tabular}{|c|c|c|c|c|}
\hline $\begin{array}{l}\text { Uniprot } \\
\text { ID }\end{array}$ & Protein name & $\begin{array}{c}\text { Fold } \\
\text { change* }\end{array}$ & p value & Functions \\
\hline P02763 & $\begin{array}{l}\text { Alpha-1-acid } \\
\text { glycoprotein } 1\end{array}$ & 3.2 & $7.49 \mathrm{E}-03$ & $\begin{array}{l}\text { Modulating the activity of the immune system } \\
\text { during the acute-phase reaction }{ }^{22,23}\end{array}$ \\
\hline P05156 & $\begin{array}{l}\text { Complement } \\
\text { factor I }\end{array}$ & 1.7 & 4.33E-02 & $\begin{array}{l}\text { Responsible for cleaving the alpha-chains of } \mathrm{C} 4 \mathrm{~b} \\
\text { and } \mathrm{C} 3 \mathrm{~b} \text { in the presence of the cofactors } \mathrm{C} 4- \\
\text { binding protein and factor } \mathrm{H} \text { respectively. }\end{array}$ \\
\hline P00734 & Prothrombin & 1.7 & $1.72 \mathrm{E}-02$ & $\begin{array}{l}\text { Thrombin, which cleaves bonds after Arg and } \\
\text { Lys, converts fibrinogen to fibrin and activates } \\
\text { factors V, VII, VIII, XIII, and, in complex with } \\
\text { thrombomodulin, protein C. Functions in blood } \\
\text { homeostasis, inflammation and wound healing. }\end{array}$ \\
\hline P02774 & $\begin{array}{l}\text { Vitamin D- } \\
\text { binding protein }\end{array}$ & 1.6 & $1.07 \mathrm{E}-03$ & $\begin{array}{c}\text { Enhancement of the chemotactic activity of C5 } \\
\text { alpha for neutrophils in inflammation and } \\
\text { macrophage activation }\end{array}$ \\
\hline
\end{tabular}

Play important roles in the innate immune response, anti-inflammatory ${ }^{24}$;

Promotes resolution of inflammation and wound healing ${ }^{25}$;

P04083 Annexin A1 - $1.7 \quad 2.58 E-07 \quad$ Promotes chemotaxis of granulocytes and monocytes $^{26}$;

Contributes to the adaptive immune response, regulates differentiation and proliferation of activated T-cells ${ }^{27}$;

\begin{tabular}{|c|c|c|c|c|}
\hline P10909 & Clusterin & -2.1 & $7.06 \mathrm{E}-04$ & Modulate NF-kappa-B transcriptional activity ${ }^{36}$ \\
\hline Q08380 & $\begin{array}{c}\text { Galectin-3- } \\
\text { binding protein }\end{array}$ & -3.0 & $1.39 \mathrm{E}-03$ & $\begin{array}{l}\text { Promotes integrin-mediated cell adhesion. May } \\
\text { stimulate host defense against viruses and tumor } \\
\text { cells. }\end{array}$ \\
\hline
\end{tabular}




\begin{tabular}{|c|c|c|c|c|}
\hline P61626 & Lysozyme C & -3.5 & $8.47 \mathrm{E}-03$ & $\begin{array}{l}\text { Associated with the monocyte-macrophage } \\
\text { system, enhance the activity of immunoreagents }\end{array}$ \\
\hline P22079 & Lactoperoxidase & -4.1 & $4.85 \mathrm{E}-04$ & $\begin{array}{l}\text { Antimicrobial agent which utilizes hydrogen } \\
\text { peroxide and thiocyanate (SCN) to generate the } \\
\text { antimicrobial substance hypothiocyanous acid } \\
\text { (HOSCN) (By similarity). May contribute to } \\
\text { airway host defense against infection. }\end{array}$ \\
\hline
\end{tabular}

$2 *$ a positive fold change indicates increased protein concentration in the relapsed eye compared 3 to the contralateral quiescent eye; a negative fold change indicates decreased protein 4 concentration in the relapsed eye compared to the contralateral quiescent eye 
Figure 1

Workflow schematic of this study. 


\section{Recruit BDU patients with unilateral relapse}

$\downarrow$

\section{Tear sample collection and preservation}

$\downarrow$

\section{Sample preparation}

$\downarrow$

\section{LC-DDA-MS/MS analysis}

$\downarrow$

\section{Spectral library building}

$\downarrow$

LC-DIA-MS/MS analysis

$\downarrow$

\section{Spectronaut Pulsar and statistical analysis}


Figure 2

Comparison of the tear proteins identified in 3 studies.

\section{This study}

Zhou L's study

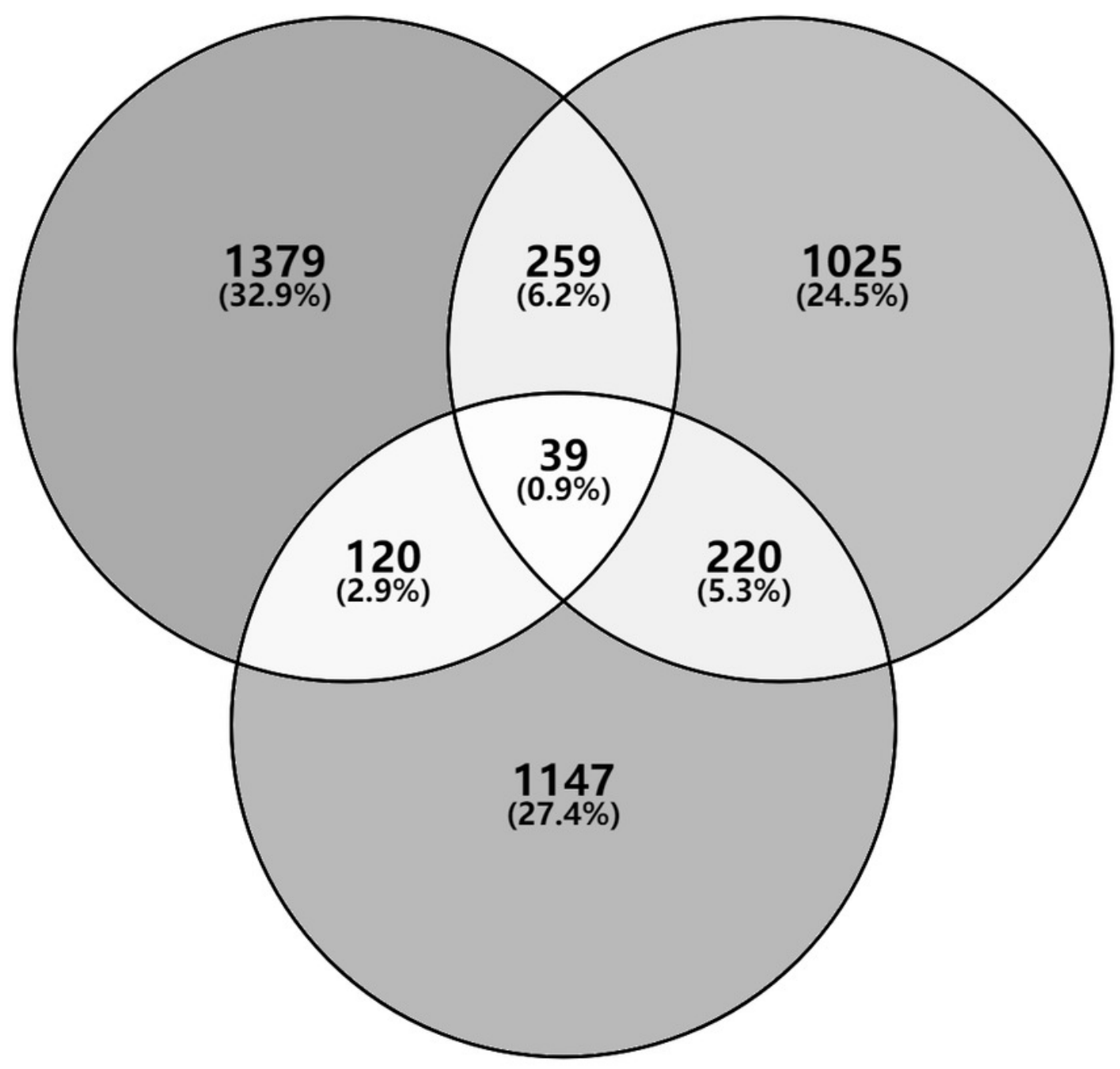

Ass C's study 